\title{
Small Bodies: Near and Far Database for thermal infrared observations of small bodies in the Solar System ${ }^{\star}$
}

\author{
Róbert Szakáts $^{1}$, Thomas Müller ${ }^{2}$, Víctor Alí-Lagoa ${ }^{2}$, Gábor Marton ${ }^{1,3}$, Anikó Farkas-Takács ${ }^{1}$, \\ Evelin Bányai ${ }^{1}$, and Csaba Kiss ${ }^{1,3}$ \\ ${ }^{1}$ Konkoly Observatory, Research Centre for Astronomy and Earth Sciences, Konkoly Thege Miklos ut 15-17, \\ 1121 Budapest, Hungary \\ e-mail: szakats.robert@csfk.mta.hu \\ 2 Max-Planck-Institut für extraterrestrische Physik, Giesenbachstrasse, Garching, Germany \\ 3 ELTE Eötvös Loránd University, Institute of Physics, Pázmány P. s. 1/A, 1171 Budapest, Hungary \\ Received 20 June 2019 / Accepted 30 December 2019
}

\begin{abstract}
In this paper, we present the Small Bodies: Near and Far Infrared Database, an easy-to-use tool intended to facilitate the modelling of thermal emission of small bodies of the Solar System. Our database collects measurements of thermal emissions for small Solar System targets that are otherwise available in scattered sources and provides a complete description of the data, including all information necessary to perform direct scientific analyses and without the need to access additional external resources. This public database contains representative data of asteroid observations of large surveys (e.g. AKARI, IRAS, and WISE) as well as a collection of small body observations of infrared space telescopes (e.g. the Herschel Space Observatory) and provides a web interface to access this data. We also provide an example for the direct application of the database and show how it can be used to estimate the thermal inertia of specific populations, e.g. asteroids within a given size range. We show how different scalings of thermal inertia with heliocentric distance (i.e. temperature) may affect our interpretation of the data and discuss why the widely-used radiative conductivity exponent $(\alpha=-3 / 4)$ might not be adequate in general, as suggested in previous studies.
\end{abstract}

Key words. astronomical databases: miscellaneous - minor planets, asteroids: general - infrared: general

\section{Introduction}

The field of analysing and modelling the thermal emission of asteroids has experienced substantial growth in the last decade, mainly thanks to the improved availability of thermal emission data (Delbó et al. 2015) and it will continue thanks to the rising

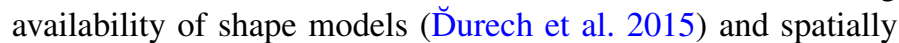
resolved in situ thermal emission data from space instruments (the Hayabusa-2 and OSIRIS-REx missions, Watanabe et al. 2017; Enos \& Lauretta 2019).

The typical subsolar temperatures of near-Earth asteroids are $\sim 300 \mathrm{~K}$, and $\sim 200 \mathrm{~K}$ in the main belt. The thermal emission of these asteroids peak in the mid-infrared $(10-20 \mu \mathrm{m})$. Although these wavelengths are partly available from the ground, the vast majority of these observations are performed by space instruments (e.g. the Akari Space Telescope and the WISE/NEOWISE surveys Usui et al. 2011; Masiero et al. 2011). Despite their warm surface temperatures, the modelling of near-Earth asteroids benefited from far-infrared observations too, such as, the thermal modelling of (101955) Bennu, (308635) $2005 \mathrm{YU}_{55}$, (99942) Apophis, and (162 173) Ryugu (Müller et al. 2012, 2013, 2014a, 2017). Beyond Jupiter and in the transneptunian region, the typical surface temperatures drop from $\sim 100 \mathrm{~K}$ to $30-50 \mathrm{~K}$ and the corresponding emission with a peak in the far-infrared $(50-100 \mu \mathrm{m})$ is almost exclusively observed from space. Centaurs and TNOs were suitably observable with the far-infrared detectors of the Spitzer Space Telescope (mostly at $70 \mu \mathrm{m}$, Stansberry et al. 2008) and Herschel Space Observatory

^ The database is available at https://ird.konkoly.hu
(70, 100 and $160 \mu \mathrm{m}$, Müller et al. 2009, 2018, 2020). Recent reviews on the thermal emission of asteroids and more distant objects by space instruments can be found in Müller et al. (2020), Mainzer et al. (2015), and references therein.

While the original primary goal of asteroid thermal infrared measurements was to derive diameters and albedos, the improvement of detector sensitivity and the availability of multi-epoch observations not only offer more precise diameters and albedos, but also allow fort the derivation of other physical properties, such as thermal inertia and surface roughness, and, consequently, such features as the porosity and physical nature of the regolith layer. Thermal emission measurements also provide information on the YORP effect (Vokrouhlický et al. 2015) and they can constrain the spin-axis orientation (see e.g. Müller et al. 2012, 2013, 2017), as well as the temperature evolution that can alter the surface or subsurface composition as well as help in shape and spin modelling (Ďurech et al. 2017), while also providing information on diurnal temperature variations that may lead to thermal cracking and, thereby, produce fresh regolith (Delbó et al. 2014).

The interpretation of the thermal emission measurements is rather complex, as the measured flux densities are strongly dependent on the epoch of the observations through the heliocentric distance of the target, the distance between the target and the observer, phase angle, aspect angle, rotational phase, and also on the thermal and surface roughness properties. The actual thermal characteristics are not fundamental properties, as, for example, the thermal inertia is a function of thermal conductivity, heat capacity, and density, while thermal conductivity and heat capacity both depend on the local temperature (see Keihm 1984; 
Keihm et al. 2012; Delbó et al. 2015; Marsset et al. 2017; Rozitis et al. 2018, and references therein). Researchers working on these types of interpretations need to collect and process all of this auxiliary information to correctly interpret the thermal emission measurements individually for all type of instruments.

The primary goal of the Small Bodies: Near and Far (SBNAF) Infrared Database (hereafter, IRDB) is to help scientists working in the field of modelling the thermal emission of small bodies by providing them with an easy-to-use tool. Our database collects available thermal emission measurements for small Solar Systems targets that are otherwise available in scattered sources and gives a complete description of the data, including all the information necessary to perform direct scientific calculations and without the need to access additional external resources. The IRDB provides disk-integrated, calibrated flux densities based on careful considerations of instrument- and project-specific calibration and processing steps. These multiepoch, multi-wavelength, multi-aspect data allow for a more complex thermophysical analysis for individual objects (e.g. using more sophisticated spin-shape solutions) or samples of objects. It will also allow for the combination of remote data with close-proximity data for the same target. In addition to answering direct scientific questions related to, for example, thermal inertia and other surface properties of the targets, it will also help in establishing celestial calibrators for instruments working in the thermal infrared regime, from mid-IR to submm wavelengths (see e.g. Müller et al. 2014b).

Early versions of the database were used in several studies. Marciniak et al. (2018a,b) performed a modelling of long-period and low-amplitude asteroids and derived the thermal properties of slowly rotating asteroids; Müller et al. (2017) obtained the spin-axis orientation of Ryugu using data from several infrared space and ground based instruments; and the 3D shape modelling and interpretation of the VLT/Sphere imaging of (6) Hebe was also supplemented by data from the infrared database (Marsset et al. 2017). Reconsiderations of the thermal emission of Haumea (Müller et al. 2019), constrained with the occultation results (Ortiz et al. 2017) was also performed using multi-epoch, multi-mission data from the SBNAF IRDB.

In the present version of the database, we included flux densities only at those wavelengths which are expected to be purely thermal, with a negligible contribution from reflected solar radiation. This excludes, for example, the two short wavelength filters of WISE, W1 and W2 (3.4 and $4.6 \mu \mathrm{m})$.

Our final aim is to include thermal data for all Solar System small bodies which have been detected at thermal infrared wavelengths. The initial version of the IRDB has been created in the framework of the Horizon 2020 project known as Small Bodies: Near and Far (COMPET-05-2015/687378, Müller et al. 2018).

Researchers working on small body thermal emission topics are encouraged to submit their own processed and calibrated thermal infrared observations to the SBNAF IRDB. These will be transformed to the database standards, supplemented with auxiliary data and made available to the planetary science community.

The structure of the paper is the following. In Sect. 2, we summarize the scheme of the SBNAF Infrared Database and give a detailed summary of its resources. In Sect. 3, we describe the auxiliary data that supplement the core data. A detailed list of the database fields and SQL examples of the possible queries are provided in Sect. 4. In Sect. 5, we investigate the impact of albedos obtained from different resources on the colour correction values we calculate as auxiliary data and we provide an example application to demonstrate the capabilities of the SBNAF
Infrared Database, and derive thermal inertias of some specific asteroid populations. Future aspects related to the database, including the submission of data to the IRDB, are discussed in Sect. 7.

\section{Thermal infrared observations of asteroids and transneptunian objects}

\subsection{Database scheme}

The main entries in our database are the (calibrated) infrared flux densities and the corresponding flux density error (denoted as $f \pm d f$ in the outline figure Fig. 1), supplemented with observational meta data - object identifier, observatory, measurement identifier, instrument-band-filter-observing mode, start and end time of the observations, duration, measured in-band flux (calibrated, aperture-/beam-corrected, non-linearity/saturationcorrected, etc.). Raw flux densities or errors and observational meta data are typically available in the catalogues or targetspecific papers where we take our basic data from. These papers/catalogues are listed in Sect. 2.2. All these flux densities are processed (e.g. converted to [Jy] from magnitudes) and brought to a common format along with all meta data in our processing (see also Fig. 1).

The final aim is to include data of near-Earth, main-belt, and transneptunian objects with significant thermal measurements from different satellite missions (IRAS, MSX, ISO, AKARI, Herschel, WISE/NEOWISE) and also from the ground. In the present, first public release, we include IRAS, MSX, Akari, Herschel, and WISE/NEOWISE observations. A more detailed description of these missions and references to available infrared data are given in Sect. 2.2. The current version of the catalogue contains 169980 entries (see Table 1).

To fully utilize these measurements, we collect auxiliary data for the observations from external sources. These data are partly stored as additional useful entries (e.g. orbital elements and coordinates from JPL/Horizons) or used to calculate quantities that are necessary for the correct interpretation of the measurements (e.g. colour correction). A list of quality comments or other relevant flags are also included. The IR database is created in such a way that all available thermal measurements of our selected targets are easily accessible through a simple web interface ${ }^{1}$.

\subsection{Key references and sources of data}

Below we give a summary of the references and sources of asteroid flux densities related to each mission and telescope. A simple reference code is also given for those papers or catalogues that presented flux densities used for our database. These codes are listed in the "documents_references" field of the database for each measurement.

IRAS. A general description of the Infrared Astronomical Satellite (IRAS) mission can be found in Neugebauer et al. (1984). A detailed summary of the IRAS mission is given in the IRAS Explanatory Supplement, available at the NASA/IPAC Infrared Science Archive ${ }^{2}$, that also covers calibration issues (point source calibration, estimated accuracy, bright source problems, colour correction). Asteroid fluxes are obtained from The Supplemental IRAS Minor Planet Survey from Tedesco et al. (2002a) [T02IRAS].

\footnotetext{
https://ird.konkoly.hu

http://irsa.ipac. caltech.edu/IRASdocs/exp.sup/
} 


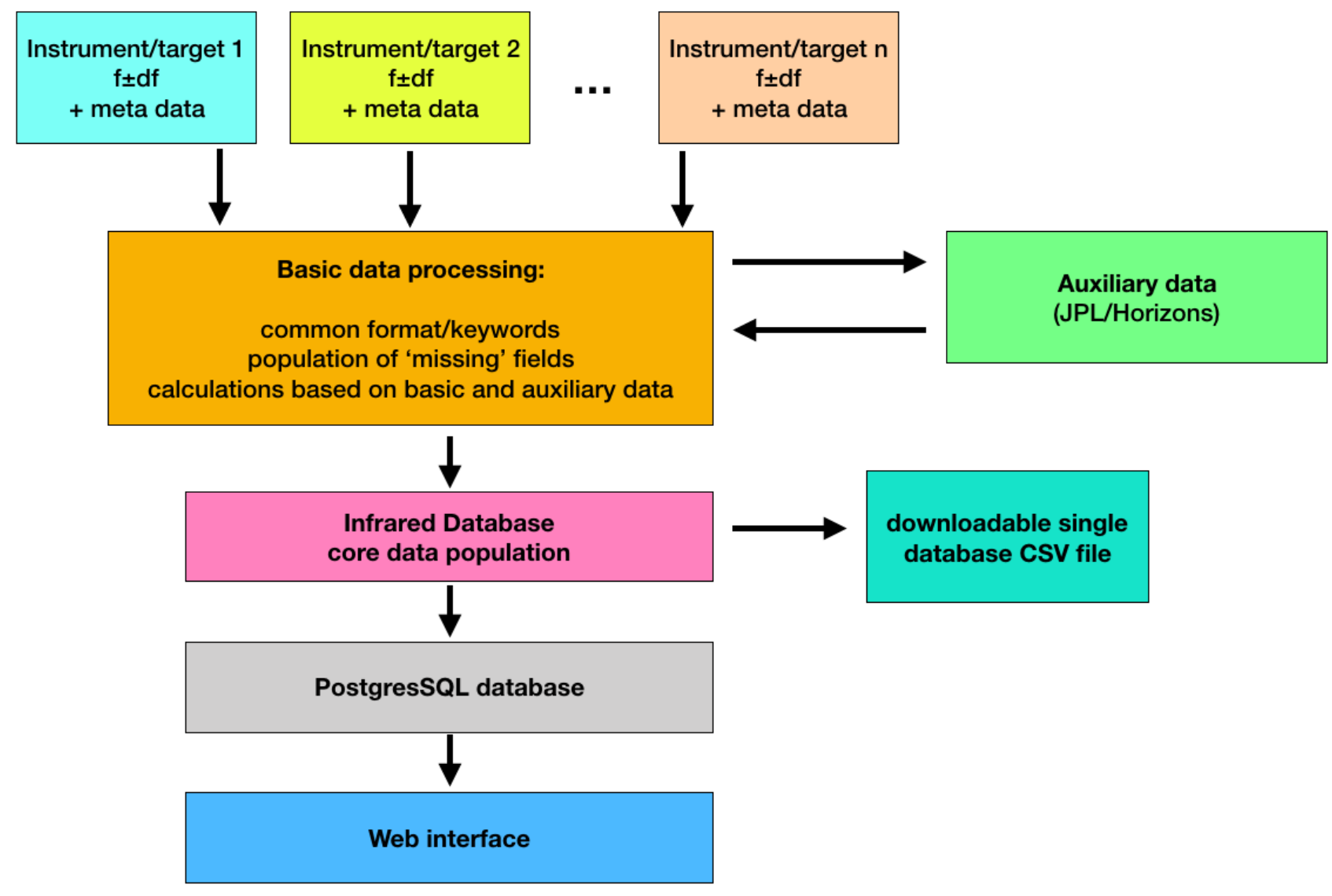

Fig. 1. Outline of the processing of data from the basic entries (catalogues and target-specific papers) to the web-interface of the SBNAF infrared database.

Table 1. Observatories or missions, instruments, filters, possible observing modes, and the number of measurements with a specific instrument, in the present version of the Infrared Database.

\begin{tabular}{lcccc}
\hline \hline Mission & Instrument & Filters & Observing mode & $N_{\text {obs }}$ \\
\hline AKARI & IRC-NIR & N4 & IRC02 & 1 \\
& IRC-MIR-S & S7, S9W, S11 & Survey, IRC02, IRC11 & 6955 \\
& IRC-MIR-L & L15, L18W, L24 & Survey, IRC02, IRC51 & 13824 \\
\hline HSO & PACS & Blue,green,red & Chop-nod, scan map & 1852 \\
\hline MSX & & MSX_A,MSX_C,MSX_D,MSX_E & Survey & 901 \\
\hline IRAS & & IRAS12,IRAS25,IRAS60,IRAS100 & Survey & 25064 \\
\hline WISE & & W3,W4 & Survey & 121383 \\
\hline
\end{tabular}

Notes. Instruments on low-Earth orbits, such as AKARI, MSX and IRAS are referred to as geocentric (JPL code "500@399”), and we used the JPL code “500@-163” for WISE and “500@-486” for Herschel.

MSX. Mill (1994) and Mill et al. (1994) provide an overview of the spacecraft, its instruments, and scientific objectives, and Price \& Witteborn (1995), Price et al. (1998) a general description of the astronomy experiments. More details on the astronomy experiments and the influence the spacecraft design has on these experiments can be found in Price et al. (2001). Asteroid fluxes are obtained from The Midcourse Space Experiment Infrared Minor Planet Survey catalogue from Tedesco et al. (2002b).

AKARI. The AKARI mission is described in Murakami et al. (2007). Minor planet flux densities are obtained from the AKARI Asteroid Flux Catalog Ver. $1^{3}$ (Release October 2016), referred

\footnotetext{
3 https://www.ir.isas.jaxa.jp/AKARI/Archive/ Catalogues/Asteroid_Flux_V1/
}

to as [AKARIAFC] in the IRDB. The catalogue contains data from the all-sky survey by Usui et al. (2011), slow-scan observation by Hasegawa et al. (2013), and pointed observations of (25 143) Itokawa and (162 173) Ryugu by Müller et al. (2014c, 2017). These "non-survey" modes have their special flags in the "obsmode" fields (IRC02: pointed observations; IRC11/IRC51: slow scan), as defined in the AKARI Asteroid Flux Catalogue.

Herschel. The Herschel Space Observatory mission is summarized in Pilbratt et al. (2010). The Photometer Array Camera and Spectrometer (PACS) instrument is described in Poglitsch et al. (2010). The photometric calibration of PACS is discussed in Nielbock et al. (2013) (chop-nod photometric observing mode) and in Balog et al. (2014) (scan-map photometric mode). 
Flux densities of Solar System small bodies are obtained from selected publications of near-Earth asteroids and Centaurs/transneptunian objects.

WISE. The WISE mission is described in Wright et al. (2010). Data products are summarized in the Explanatory Supplement to the AllWISE Data Release Products (Cutri et al. 2013).

The WISE Moving Object Pipeline Subsystem (WMOPS) reported all detections of Solar System small bodies to the IAU Minor Planet Center (MPC) for confirmation, whereas the computed in-band magnitudes were collected in the IRSA/IPAC archive, namely in the Level $1 \mathrm{~b}$ catalogues. To retrieve these magnitudes, we queried the IPAC archive using a 1 arcsec cone search radius around the MPC-reported tracklets, which are all labelled "C51" by the MPC. In this way, we avoid using false detections that may have been included in the IPAC archive (Mainzer et al. 2011).

Since we are only interested in flux densities collected during the fully-cryogenic phase of the mission, we queried the WISE All-Sky Database. The in-band magnitudes $(m)$ were converted to in-band flux densities $(\langle f\rangle)$ as:

$\langle f\rangle=\left\langle f_{0}\right\rangle 10^{-0.4 m}$,

where $\left\langle f_{0}\right\rangle$ is the zero-magnitude isophotal flux density of Vega for each band, as reported in Wright et al. (2010). By definition, $\left\langle f_{0}\right\rangle$ does not require a colour correction. From the tabulated magnitude error bar $\Delta m$, the corresponding error bar of the in-bad flux is given by:

$\Delta f=0.40 \log _{10}\langle f\rangle \Delta m$.

To correct for a discrepancy between red and blue calibrators observed after launch, Wright et al. (2010) suggest shifting the W3 and W4 isophotal wavelengths and correcting the isophotal flux densities accordingly. Thus, we took 11.10 and $22.64 \mu \mathrm{m}$ and 31.368 , and 7.952 Jy, respectively (more details in Masiero et al. 2011). Flux densities obtained using this procedure are referred to as [WISEASD] in the respective field of our IRDB. In this release of the database, we included only those WISE measurements where there is also AKARI data for that object.

\section{Auxiliary data}

In our database, in addition to the basic data available in the resources listed above (measured (in-band) flux density and its uncertainty, date, instrument and filter, etc.), each measurement is supplemented with additional (auxiliary) data, either with data from external resources (mainly JPL/Horizons, Sect. 3.1), as well as values calculated from other fields of the database. The latter include the calculation of the monochromatic flux density at a pre-defined reference wavelengths (colour correction), conversion of calendar date to Julian date (JD, with or without correction for light-travel time), and adding absolute flux density errors (Sect. 3.2). The procedures to obtain these auxiliary data are summarized below.

\subsection{JPL Horizons data}

Our database uses data obtained from NASA's JPL-Horizons service. These data are stored in the database and available directly in the IRDB and, in some cases, also used for further calculations. We query the following parameters from JPL-Horizons:

- Orbital elements: semi-major axis, a (AU); eccentricity, e; inclination w.r.t $X Y$-plane, $i\left(^{\circ}\right)(X Y$-plane: plane of the Earth's orbit at the reference epoch ${ }^{4}$; longitude of ascending node, $\Omega\left(^{\circ}\right)$; argument of perifocus, $\varpi\left(^{\circ}\right)$; mean anomaly, $M\left(^{\circ}\right)$.

- Parameters related to size and albedo: absolute magnitude, $H$ (mag); slope parameter $G$; object's effective radius $[\mathrm{km}]$; object's $V$-band geometric albedo.

- Apparent position: apparent right ascension (RA) and declination (Dec) at the time of the observation, ICRF/J2000 inertial reference frame, compensated for down-leg light-time [deg/deg]; rate of change of target center apparent RA and Dec (airless). We note that $\mathrm{dRA} / \mathrm{d} t$ is multiplied by the cosine of the declination [both in $\operatorname{arcsec} \mathrm{h}^{-1}$ ].

- Target brightness: asteroid's approximate apparent visual magnitude and surface brightness,

$\mathrm{AP}_{\text {mag }}=H+5 \log _{10}(\Delta)+5 \log _{10}(r)-2.5 \log _{10}\left((1-G) \Phi_{1}+G \Phi_{2}\right)$,

where $H$ is the absolute brightness, $r$ the heliocentric distance of the target, $\Delta$ is the observer distance, and $G, \Phi_{1}$ and $\Phi_{2}$ are the slope parameter and the two base functions in Bowell et al. (1989) [mag, mag $\operatorname{arcsec}^{-2}$ ].

- Heliocentric distance: heliocentric range ( $r$, light-time corrected) and range-rate ("rdot") of the target center [AU and $\mathrm{km} \mathrm{s}^{-1}$ ]. In addition, the one-way down-leg light-time from target center to observer is also retrieved [s].

- Sun-Observer-Target angle: target's apparent solar elongation seen from the observer location at print-time $\left[{ }^{\circ}\right]$. " $/ \mathrm{r}$ " flag indicating the target's apparent position relative to the Sun in the observer's sky (“/T” for trailing, "/L" for leading).

- Ecliptic coordinates of the target: Observer-centered Earth J2000.0 ecliptic longitude and latitude of the target center's apparent position, adjusted for light-time, the gravitational deflection of light and stellar aberration [deg/deg].

- $X, Y, Z$ Cartesian coordinates of the target body and observer/Earth at the time of observation in the J2000.0 reference frame defined in Archinal et al. (2011) [AU].

Data from JPL Horizons are obtained by four functions in the main python script. These functions are:

- get_jplh: this function obtains the ephemeris data from JPL Horizons, such as RA, Dec, $r$, delta, etc.

- get_jplhelements: the orbital elements of the target body are retrieved with this function.

- getvecs1 \& getvecs2: these functions obtain the $X, Y, Z$ position vectors from JPL with respect to the Sun and the observer.

The following parameters are also extracted from the .csv files obtained by these functions: $H$, JPL radius, albedo, and slope parameter. In those cases when JPL recognizes the target as a comet and not an asteroid, $T$-mag (Comet's approximate apparent visual total magnitude), $M 1$ (Total absolute magnitude) parameters are extracted and used. Also, a comment is written into to the database in this case.

The functions use astropy.table from Astropy (Astropy Collaboration 2013, 2018) and urllib3 ${ }^{5}$.

\subsection{Calculated values}

Obtaining monochromatic flux density (colour correction). For most of the instruments or filters included in the IRDB, colour correction is calculated using the relative response profiles of the specific filters and assuming an estimated effective

\footnotetext{
4 Obliquity of 84381.448 arcsec with respect to the ICRF equator (IAU76).

5 https://pypi.org/project/urllib3/
} 
Table 2. Resources of Herschel Space Observatory measurements.

\begin{tabular}{|c|c|c|}
\hline \multicolumn{3}{|l|}{ Near-Earth asteroids } \\
\hline Müller et al. (2012) & [M12] & (101955) Bennu \\
\hline Müller et al. (2017) & [M13] & (308625) 2005 YU55 \\
\hline Müller et al. (2017) & [M14] & (99942) Apophis \\
\hline Müller et al. (2017) & [M17] & (162 173) Ryugu \\
\hline \multicolumn{3}{|c|}{ Centaurs and transneptunian objects } \\
\hline Duffard et al. (2014) & [D14] & 16 Centaurs \\
\hline Fornasier et al. (2013) & {$[\mathrm{F} 13]$} & $\begin{array}{l}\text { (2060) Chiron, (10 199) Chariklo, (38 628) Huya, (50 000) Quaoar, } \\
\text { (55 637) 2002 UX25, (84 522) 2002 TC302, (90 482) Orcus, (120347) } \\
\text { Salacia, (136 108) Haumea }\end{array}$ \\
\hline Kiss et al. (2013) & {$[\mathrm{K} 13]$} & 2012 DR30 \\
\hline Lellouch et al. (2013) & {$[\mathrm{L} 10]$} & (136 108) Haumea \\
\hline Lellouch et al. (2013) & [L13] & 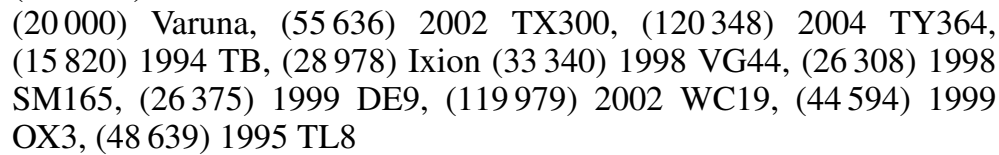 \\
\hline Lellouch et al. (2016) & [L16] & Pluto+Charon \\
\hline Lim et al. (2010) & [LIM10] & (136 472) Makemake, (90 482) Orcus \\
\hline Mommert et al. (2012) & [MM12] & 18 plutinos \\
\hline Müller et al. (2010) & [M10] & $\begin{array}{l}(208996) 2003 \text { AZ84, }(126154) 2001 \text { YH140, }(79360) \text { Sila- } \\
\text { Nunam, (82 075) 2000 YW134, (42355) Typhon, } 2006 \text { SX368, } \\
(145480) 2005 \text { TB190 }\end{array}$ \\
\hline Müller et al. (2020) & [M19A] & (136 081) Haumea \\
\hline Pál et al. (2012) & {$[\mathrm{P} 12]$} & (90 377) Sedna, 2010 EK 139 \\
\hline Pál et al. (2015) & {$[\mathrm{P} 15]$} & 2013 AZ 60 \\
\hline Santos-Sanz et al. (2012) & [SS12] & 15 scattered disk and detached objects \\
\hline Santos-Sanz et al. (2017) & [SS17] & (84 922) 2003 VS 2, (208 996) 2003 AZ 84 \\
\hline Vilenius et al. (2012) & [V12] & 19 classical transneptunian objects \\
\hline Vilenius et al. (2014) & [V14] & 18 classical transneptunian objects \\
\hline Vilenius et al. (2018) & [V18] & $\begin{array}{l}1995 \text { SM 55, } 2005 \text { RR 43, } 2003 \text { UZ 117, } 2003 \text { OP 32, } 2002 \text { TX 300, } \\
1996 \text { TO 66, } 1999 \text { CD 158, } 1999 \text { KR } 16\end{array}$ \\
\hline
\end{tabular}

temperature $\left(T_{\text {eff }}\right)$ for the target, which is calculated as:

$T_{\mathrm{eff}}=\frac{393.6 \mathrm{~K}}{\sqrt{r_{\mathrm{h}}}}\left(1-p_{V} \cdot q\right)^{1 / 4}$,

where $r_{\mathrm{h}}$ is the heliocentric distance (AU), $p_{V}$ is the $V$-band geometric albedo and $q$ is the standard Bowell et al. (1989) phase integral: $q=0.290+0.684 \mathrm{G}$, where $G$ is the slope parameter and using the solar constant of $1361.5 \mathrm{~W} \mathrm{~m}^{-2}$ (Kopp \& Lean 2011) and the Stefan-Boltzmann constant to get $T=393.6 \mathrm{~K}$ at $1 \mathrm{AU}$. We assumed an emissivity of unity. We use $T_{\text {eff }}$ to calculate a black body SED which we utilize during the colour correction. But in case of high-albedo objects this formula does not work well (see Vilenius et al. 2018 and Brucker et al. 2009). If $p_{V}$ is unknown or uncertain, we use $p_{V}=0.10$ (see Sect. 5). We note that the $p_{V}$ in Eq. (4) is obtained from the tabulated $H$ and $D$ values from the Horizons JPL service. Arguably, there are other sources of diameters and $H$ values that could be used to calculate $p_{V}$ but the colour correction values are not extremely sensitive to large variations of $p_{V}$ (see Sect. 5 for further discussion of this point). We include these diameters, albedos, and $H-G$ values in the database for the sake of reproducibility of the monochromatic flux densities, but for statistical analyses with these quantities we recommend using or at least examining other sources (e.g. Delbó et al. 2017 ${ }^{6}$; Oszkiewicz et al. 2011; Vereš et al. 2015). The colour corrected or monochromatic flux density

\footnotetext{
6 https://www-n.oca.eu/delbo/astphys/astphys.html
}

is obtained as $f_{\lambda}=f_{i} / K(\lambda)$, where $f_{i}$ is the in-band flux density obtained directly from the measurements and $K(\lambda)$ is the colour correction factor, which is obtained using the spectral energy distribution of the source (flux density $F_{v}(\lambda)$ ) and the relative response of the detector/filter system $\left(R_{v}(\lambda)\right)$ as:

$$
K(\lambda)=\frac{\frac{1}{F_{v}\left(\lambda_{\mathrm{c}}\right)} \int F_{v}(\lambda) R_{v}(\lambda) \mathrm{d} \lambda}{\frac{1}{F_{v}^{\mathrm{ref}}\left(\lambda_{\mathrm{c}}\right)} \int F_{v}^{\mathrm{ref}}(\lambda) R_{v}(\lambda) \mathrm{d} \lambda},
$$

where $\lambda_{\mathrm{c}}$ is the central (reference) wavelength of the filter in the photometric system and $F_{v}^{\text {ref }}(\lambda)$ is the reference spectral energy distribution of the photometric system (typically $v F_{v}=$ const.).

The monochromatic flux density uncertainties are calculated as:

$\delta f_{\lambda}=\sqrt{\frac{1}{K(\lambda)^{2}}\left[\Delta f_{i}^{2}+\left(r_{\mathrm{abs}} f_{i}\right)^{2}\right]+\left(r_{\mathrm{cc}} f_{\lambda}\right)^{2}}$,

where $\Delta f_{i}$ is the in-band flux density uncertainty and $r_{\mathrm{abs}}$ is the absolute calibration error, usually expressed as a fraction of the in-band flux (see below). The last term contains the flux density uncertainty due to the colour correction uncertainty characterised by $r_{\mathrm{cc}}$, which is approximately proportional to the deviation of the actual value of the colour correction from unity. In the present version, it is implemented in the following way:

- if $0.95 \leq K(\lambda) \leq 1.05$ then $r_{\mathrm{cc}}=0.01$

- if $0.90 \leq K(\lambda) \leq 0.95$ then $r_{\mathrm{cc}}=0.02$

- if $K(\lambda) \leq 0.90$ or $K(\lambda) \geq 1.05$ then $r_{\mathrm{cc}}=0.03$. 


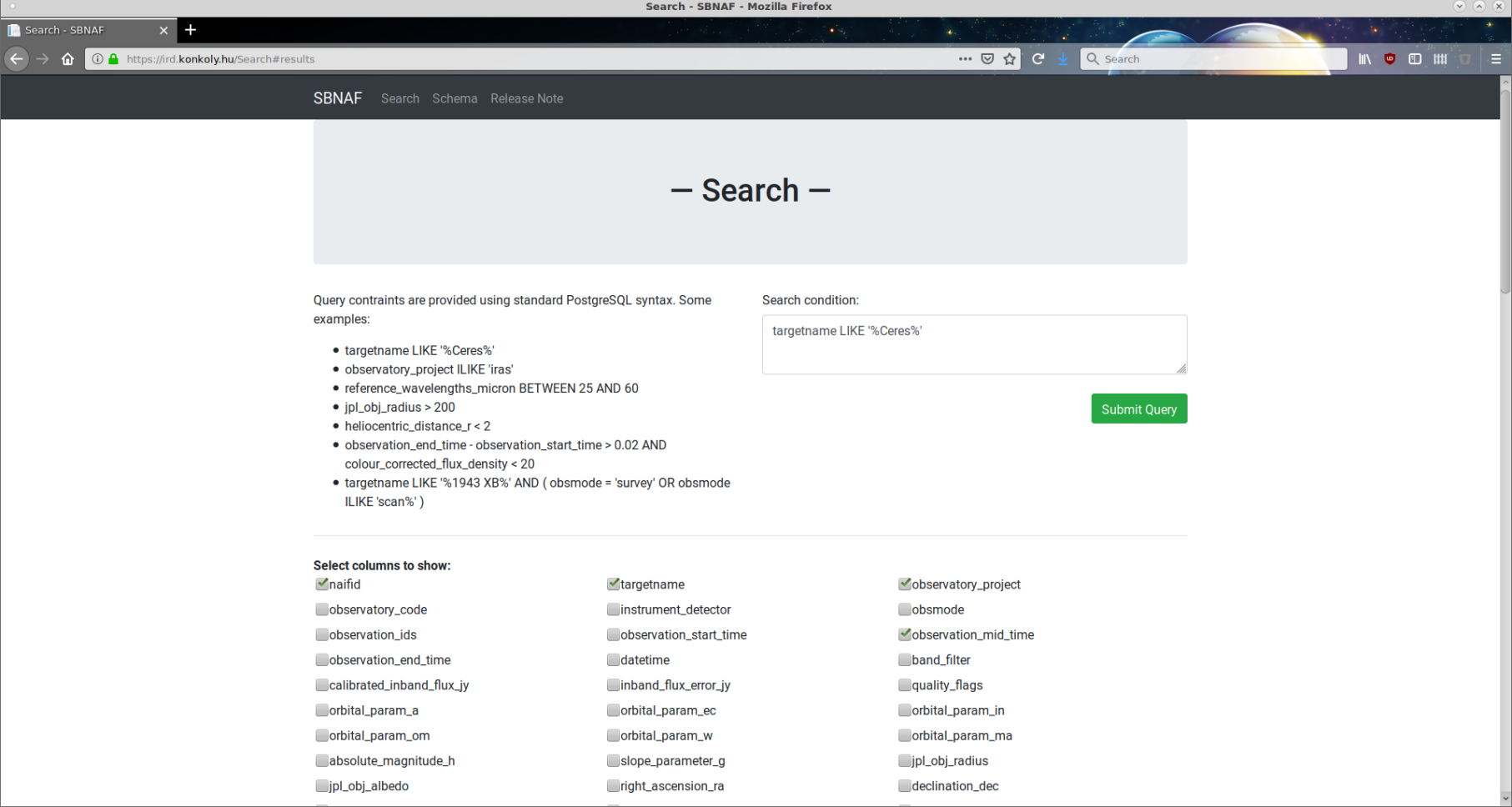

Fig. 2. Query of 1 Ceres, with some examples on the left.

Procedure for adding the absolute calibration error. Absolute calibration error is calculated as described above in Eq. (6). The $r_{\text {abs }}$ factor is instrument- or filter-dependent and it is determined during the flux calibration of the instrument and is described in instrument specific calibration papers. These $r_{\mathrm{abs}}$ values are following:

- IRAS: $10 \%, 10 \%, 15 \%$ and $15 \%$ at 12, 25, 60 and $100 \mu \mathrm{m}$ (Tedesco et al. 2002a)

- Herschel/PACS: 5\%, 5\% and 7\% at 70, 100 and $160 \mu \mathrm{m}$ (Balog et al. 2014)

- WISE: 5\% in all bands (Wright et al. 2010; Rozitis et al. 2014; Hanuš et al. 2015)

- MSX $^{7}: 5 \%, 5 \%, 6 \%$ and $6 \%$ at $8.28,12.13,14.65$ and $21.34 \mu \mathrm{m}$ (Egan et al. 2003)

- AKARI: 5\% in all bands (Usui et al. 2011; Alí-Lagoa et al. 2018).

\section{Database and access}

\subsection{Main database file and web interface}

From the collected data (flux densities, observational meta data, and auxiliary data, see above) a PostgreSQL table is created that is the essentially the SBNAF Infrared Database. The database is accessible through a web interface ${ }^{8}$ (at Konkoly Observatory). An example is shown below, presenting the query screen (Fig. 2) and the resulting output screen (Fig. 3) for some selected IRAS and Akari observations of 1 Ceres.

The SBNAF IRDB webpage is running on a dedicated linux machine. The webserver is NGINX and the webpage itself is a ASP.NET Core Web Application, which uses the ASP.NET

\footnotetext{
7 We took the absolute calibration uncertainties from Table 1 except for band $A$, for which we used a more conservative $5 \%$ absolute calibration error bar.

8 Available at https://ird.konkoly.hu
}

Core 2.0 Framework. This web application communicates with the webserver, with the users through the webpage and with the database itself (PostgreSQL table). The web content is generated on the server, and the users can only see the static HTML page in their web browser at the end. The user has the option to download the output page as a .csv file (using the "Download" button).

The example above was generated by the following query: targetname LIKE “\% Ceres\%" and (observatory_project LIKE “IRAS" or observatory_project ILIKE “Akari”), requiring exact, case sensitive matching with "LIKE" on "targetname" (="Ceres") and selecting observatories "IRAS" (exact name again with "LIKE") and "Akari" (selecting with "ILIKE", i.e. case-insensitive). In this case, the output gives the default selection: "naifid", "targetname", "observatory_project", "observation_mid_time", “colour_corrected_flux_density", and "absolute_flux_error”.

Numeric data types (FLOAT, DOUBLE) can be queried as follows: observation_start_time BETWEEN 2453869 AND 2454000: selects the objects where the observation time started between 2453869 and 2454000 (JD); observation_start_time > 2453869 AND observation_start_time $<\mathbf{2 4 5 4 0 0 0}$ : returns the same results as the previous query. naifid $=\mathbf{2 0 0 0 0 0 1}$ : returns all targets with "naifid" exactly equal to 2000001.

For string type data: targetname ILIKE "\%ceres\%": selects the targets with the substring "ceres" in it, case insensitive; observatory_project $=$ "IRAS": returns all observations where the observatory_project name matches "IRAS" exactly.

The query "comments_remarks ILIKE “\%comet\%"” lists measurements of comets (currently the Akari observations of the comet P/2006 HR30 (Siding Spring) in the D2.5 version of the database).

Additional examples are given on the starting page of the web interface. The summary of the database fields is given in the appendix. 


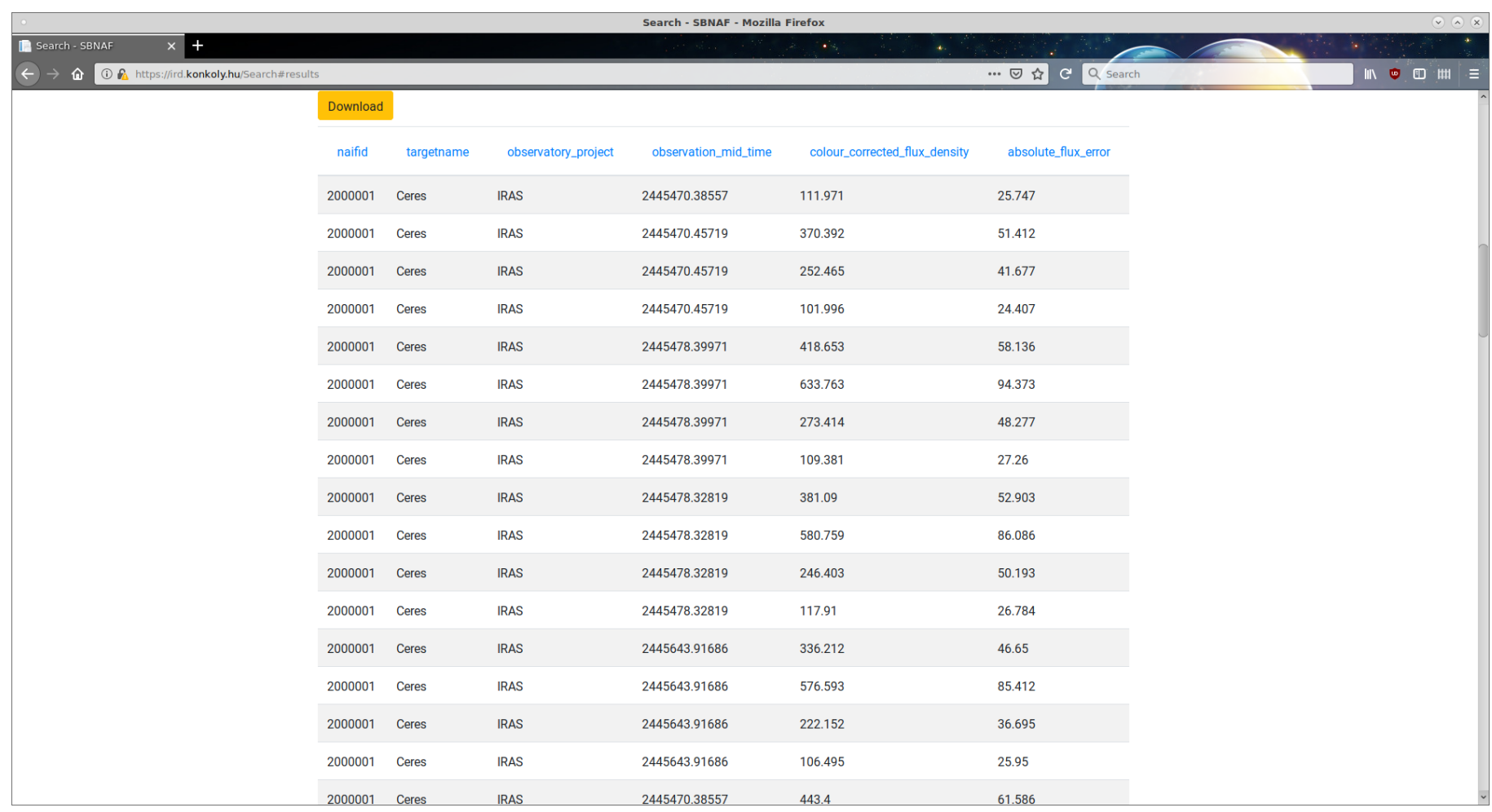

Fig. 3. Results listing infrared observation of 1 Ceres and displaying the default output selection: "naifid", "targetname", "observatory_project", "observation_mid_time", “colour_corrected_flux_density", and "absolute_flux_error".

\subsection{Alternative access}

For those who want a more scriptable form of access to the IRDB, we will upload the database into the VizieR catalog service (Ochsenbein et al. 2000). This database is widely used by the community and it provides an interface to search trough the different databases, and it can be queried trough several tools, like Astropy's astroquery.vizier interface or Topcat. In case of a major update we will upload a new data release to VizieR.

Also, we began the integration of the IRDB to the VESPA VO service ${ }^{9}$ (Erard et al. 2018). In this way our database can be accessed via EPN-TAP protocol, which is well known in the Virtual Observatory community. VESPA also provides a great tool for searching trough the different data services and with its help, the IRDB can become more accessible to researchers working on topics related to small body thermal emission. Because the VEASPA service will use the same database as our webpage, the data entered there will be always up to date.

The whole database can be downloaded directly from our webpage in csv format. The datafile contains a header and exactly the same data that can be accessed via the SQL query form. The size of the raw csv file is $\sim 125 \mathrm{MB}$ in the current release.

\section{Albedos and colour corrections of transneptunian objects}

As described in Sect 3.1, albedo information is taken by default from the NASA/Horizons service. In case these albedo values are not known, we assumed an intermediate value of $p_{V}=0.10$ to calculate the colour correction, which is a reasonable

\footnotetext{
9 https://voparis-wiki.obspm.fr/display/VES/EPN-TAP+
} Services approximation for objects with albedos $p_{V} \lesssim 0.30$. We investigated how different the colour correction factors obtained using the Horizons albedo values are from those obtained using albedos calculated for specific objects based on radiometry in previous studies (e.g. Mommert et al. 2012; Santos-Sanz et al. 2012; Vilenius et al. 2012, 2014). This comparison was performed for Centaurs and transneptunian objects, as in these cases significant deviations are expected from the general, low albedo values.

Figure 4 presents the colour correction factors obtained using the albedo values from NASA/Horizons (top row), as well as those obtained from specific publications (bottom row). In general, the differences in the colour correction factors are negligible (mainly because the corrections factors typically close to 1 in the surface temperature range for the Herschel/PACS filters). However, for some individual targets (e.g. those with large heliocentric distance and relatively high albedo) the colour correction factors may differ by $5-10 \%$. Therefore, in the updated version of the infrared database, we used the object-specific values whenever they are available.

\section{Thermal inertias of asteroid populations}

In this section, we provide an example of how the database can be used to study scientific questions. Namely, we show how to explore the dependence of thermal inertia $(\Gamma)$ with temperature $(T)$ or, equivalently, heliocentric distance $(r)$, and how to compare populations of asteroids with different sizes. These quantities are relevant, for example, for studies of dynamical evolution where the Yarkovsky effect plays a role since it is influenced among other factors by $\Gamma$ (see e.g. Bottke et al. 2006).

Thermal inertia is defined as $\Gamma=\sqrt{\rho C_{\kappa}}$, where $\rho$ is the density, $C$ the specific heat capacity, and $\kappa$ the thermal conductivity of the material. Its SI unit is $\mathrm{J} \mathrm{m}^{-2} \mathrm{~s}^{-1 / 2} \mathrm{~K}^{-1}$. Most thermo-physical models of asteroids used so far to constrain $\Gamma$ 

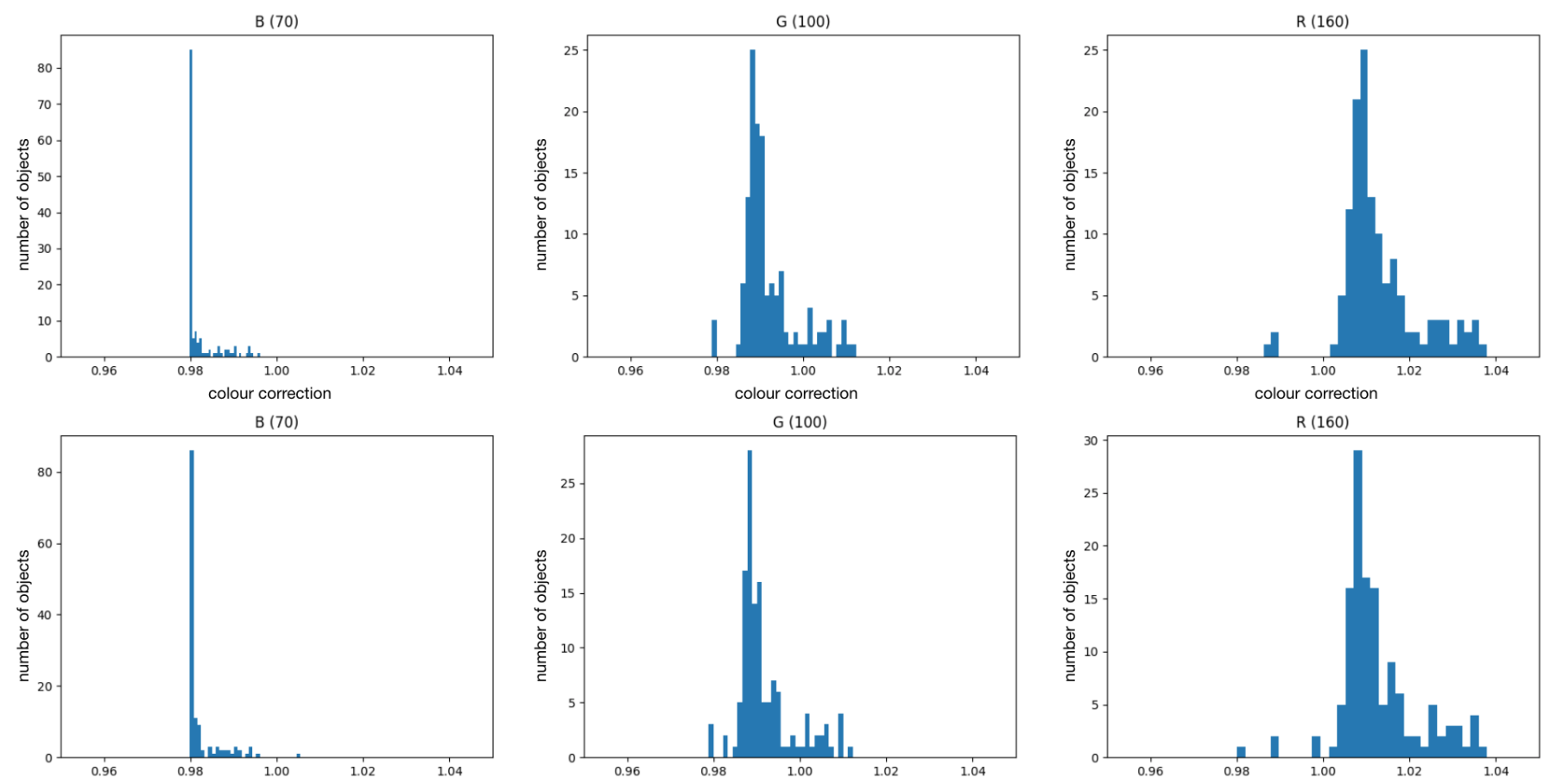

Fig. 4. Comparison of the colour correction factors obtained using the "default" albedo data from NASA/Horizons (upper row), and the albedos obtained from specific publications and/or processing. B(70), G(100) and $\mathrm{R}(160)$ mark the 70, 100 and $160 \mu \mathrm{m}$ filters of the PACS camera.

have assumed it is constant even though the heat conductivity is itself a function of temperature. For this reason, thermal inertias determined from observations taken at different heliocentric distances cannot be compared directly and must be normalised to an agreed heliocentric distance (see, e.g. Delbó et al. 2015 for a review). Assuming that heat is transported through the regolith mainly by radiative conduction within the spaces between the grains (Jakosky 1986; Kuhrt \& Giese 1989), we have $\kappa \propto T^{3}$ and hence $\Gamma \propto r^{-3 / 4}$ (see also the discussion by Delbó et al. 2007). We can then write

$\Gamma(r)=\Gamma_{0} r^{\alpha}$,

where $\Gamma_{0}$ is the value at $1 \mathrm{au}, r$ is expressed in au, and $\alpha=-3 / 4$ is the classically-assumed radiative conductivity exponent.

Rozitis et al. (2018) studied three eccentric near-Earth asteroids observed by WISE more than once at a wide range of $r$ and showed that $-3 / 4$ may not be appropriate in general. Instead, they found that each one of their targets required different exponents to fit their $\Gamma$-vs. $-r$ plots, namely $\alpha=-2.2,-1.5$, and -0.92 , although only the error bars of the first case, (1036) Ganymed, were incompatible with the "classical" $\alpha=-3 / 4$. This effect requires further studies. Below, we propose ways to use our database to examine how $\alpha$ affects our interpretation of the thermal IR observations.

With our database, we can readily select the thermally dominated WISE W3 and W4 fluxes of a group of asteroids within a given size and albedo range and compare how models with different pairs of values of $\Gamma$ and $\alpha$ fit the observations. First, we took 10-km, low-albedo asteroids. To account for uncertainties, we queried the database for all W3 and W4 colour-corrected fluxes of objects in the size range $9 \mathrm{~km} \leq D \leq 11 \mathrm{~km}$ with visible geometric albedos $p_{V} \leq 0.12$. This sample includes $\sim 4000$ fluxes per band, corresponding to about 300 asteroids, the vast majority of which are main-belt asteroids. Figure 5 shows these fluxes plotted against heliocentric distance at the epoch of observations. For reference, we also show the fluxes at which the onset of saturation occurs in each band with horizontal lines of the corresponding colour.

Next, we used the thermo-physical model of Delbó et al. (2007) under the Lagerros approximation (Lagerros 1996, 1998) to calculate the W3 and W4 fluxes at various heliocentric distances $(r=1.25,1.5,2.0,2.5, \ldots 6.0 \mathrm{au})$ for a rotating sphere ${ }^{10}$ with rotation period $P=6 \mathrm{~h}$,

Bond albedo $A=0.03$, surface roughness $\mathrm{rms}=0.6$ (the default value for main belt asteroids chosen by Müller \& Lagerros 1998; Müller 2002), and different thermal inertias at 1 au. We sampled $\Gamma_{0}=10,30,50,100,150,200 \mathrm{~J} \mathrm{~m}^{-2} \mathrm{~s}^{-1 / 2} \mathrm{~K}^{-1}$ and $\alpha=0,-0.75$ and -2.20 , and used Eq. (7) to compute the corresponding $\Gamma(r)$ for each case.

Figures 5-7 show the W3 and W4 data and the best fitting models corresponding to $\alpha=0,-0.75$, and -2.20 . The best $\Gamma_{0}$ in each case increases with $\alpha$, namely 10,50 and $150 \mathrm{~J} \mathrm{~m}^{-2} \mathrm{~s}^{-1 / 2} \mathrm{~K}^{-1}$. These models appear to reproduce the data trend reasonably well considering the numerous approximations that we made. To establish a quantitative comparison, we averaged the data within 0.5 au-wide heliocentric distance bins centred at $2.0,2.5, \ldots 4.5 \mathrm{au}$, and used these averages $\left(f_{\text {bin }}\right)$ and standard deviations $\left(\sigma_{\text {bin }}\right)$ to calculate the $\chi^{2}$ for each model $\left(F_{\text {bin }}\right)$ as $\chi^{2}=\sum_{\text {bins }}\left[\left(F_{\text {bin }}-f_{\text {bin }}\right) / \sigma_{\text {bin }}\right]^{2}$. Considering two degrees of freedom, $\alpha$ and $\Gamma_{0}$, all three cases produced reduced $\chi^{2}$ between $\sim 4.5$ and 5 indicating that the data are fit at 2.2 sigma only in average. Hence, we cannot simultaneously optimise $\alpha$ and $\Gamma_{0}$ with this approach given the current lack of "groundtruth" information for objects in this size range.

Nonetheless, it is still worth putting these models in the context of other populations. For example, if $\alpha=0$ was to be confirmed, we would conclude that the typical $\Gamma_{0}$ 's of $10-\mathrm{km}$

${ }^{10}$ More specifically, we averaged the fluxes of a prograde sphere and a retrograde sphere with pole ecliptic latitudes of $\pm 90^{\circ}$, respectively. In addition, to reproduce WISE's observation geometry, the model fluxes are computed for an observer-sun-asteroid configuration in quadrature assuming a circular orbit on the ecliptic. 


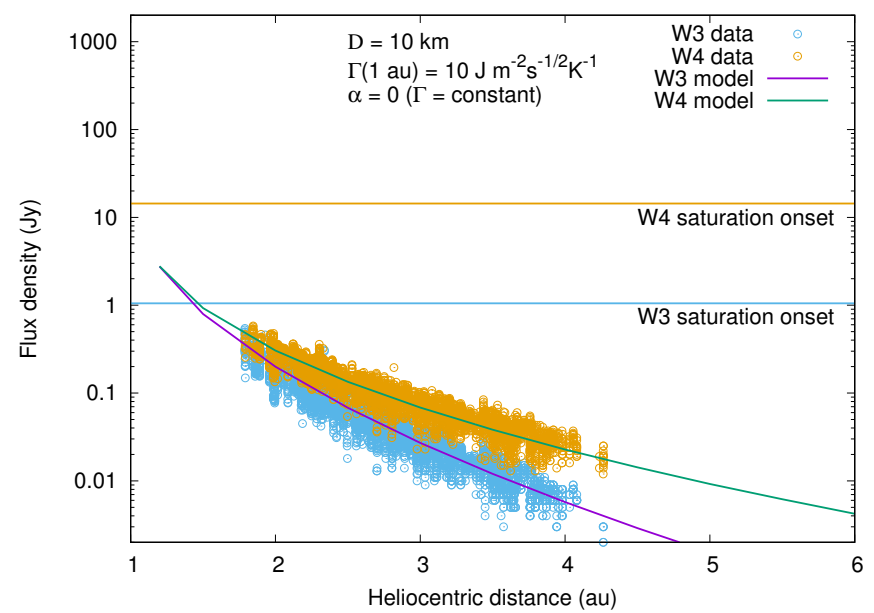

Fig. 5. WISE W3 and W4 colour-corrected fluxes of 10-km asteroids vs. heliocentric distance (blue and ochre empty circles). The purple and green lines are the corresponding model fluxes with $\Gamma_{0}=$ $10 \mathrm{~J} \mathrm{~m}^{-2} \mathrm{~s}^{-1 / 2} \mathrm{~K}^{-1}$ and $\alpha=0$. For reference, since we have excluded partially saturated fluxes from the analysis (cf. Fig. 8, left panel), we also indicate the fluxes at which saturation occurs in each band (Cutri et al. 2012).

asteroids would be a factor of 5 lower than that of the Moon's $\left(\sim 50 \mathrm{~J} \mathrm{~m}^{-2} \mathrm{~s}^{-1 / 2} \mathrm{~K}^{-1}\right)$. This would be unexpected because so far such low $\Gamma$ s have only been found among the largest asteroids (e.g. Müller 2002; Mueller et al. 2006; O'Rourke et al. 2012; Delbó \& Tanga 2009; Hanuš et al. 2018), but we cannot rule the possibility out from this approach alone. Among the models with $\alpha=-3 / 4$, the best $\Gamma_{0}$ is very close to the lunar value and the average $\Gamma_{0}$ of the nine 10 -km objects in Table A.3 of Hanus et al. (2018), namely $\sim 58 \mathrm{~J} \mathrm{~m}^{-2} \mathrm{~s}^{-1 / 2} \mathrm{~K}^{-1}$. However, this does not confirm $\alpha=-3 / 4$ as the best value for $10-\mathrm{km}$ either. Based on previous modelling reviewed by Delbó et al. (2015); see their right panel of Fig. 9; we would actually expect higher $\Gamma_{0} \mathrm{~s}$ for the "classical" $\alpha$ value, specifically in the $\sim 300 \mathrm{~J} \mathrm{~m}^{-2} \mathrm{~s}^{-1 / 2} \mathrm{~K}^{-1}$ (the role of size is further discussed below). A larger sample of $10-\mathrm{km}$ asteroids with accurate shapes and modelled $\Gamma \mathrm{s}$ would be needed to establish a more conclusive result. We would also need many more high-quality shape models of highly eccentric objects to enable additional studies like Rozitis et al. (2018) in order to constrain $\alpha$, given its crucial impact on the $\Gamma_{0}$ values and ultimately on their physical interpretation, especially in the light of the results from the space missions.

The approach illustrated here does enable the comparison of thermal inertias of differently sized populations if we keep all other parameters equal. For instance, we have about 2500 observations of the $\sim 100$ objects with sizes of $75 \mathrm{~km}$ that are featured both in the WISE and the AKARI catalogues (see Sec. 2.2). With $\alpha=-2.20$, we require a lower $\Gamma_{0}$ to fit 75 $\mathrm{km}$ asteroid data $\left(50 \mathrm{~J} \mathrm{~m}^{-2} \mathrm{~s}^{-1 / 2} \mathrm{~K}^{-1}\right)$ than $10-\mathrm{km}$ asteroid data $\left(150 \mathrm{~J} \mathrm{~m}^{-2} \mathrm{~s}^{-1 / 2} \mathrm{~K}^{-1}\right)$. The W3 and W4 (left panel) and S9W and L18W (right panel) data and corresponding model fluxes for 75-km asteroids are shown in Fig. 8. Although there are fewer objects in this size range and removing partially saturated data introduces an artefact in the W3 data trend, the conclusion that smaller asteroids have higher thermal inertias is robustly supported, at least when comparing the $10-75-\mathrm{km}$ diameter range.

Finally, some of the effects not accounted for by our simplifying assumptions could be added into the modelling in future work, such as, a large set of shapes with different elongations or degrees of irregularity and spin pole orientations. With these,

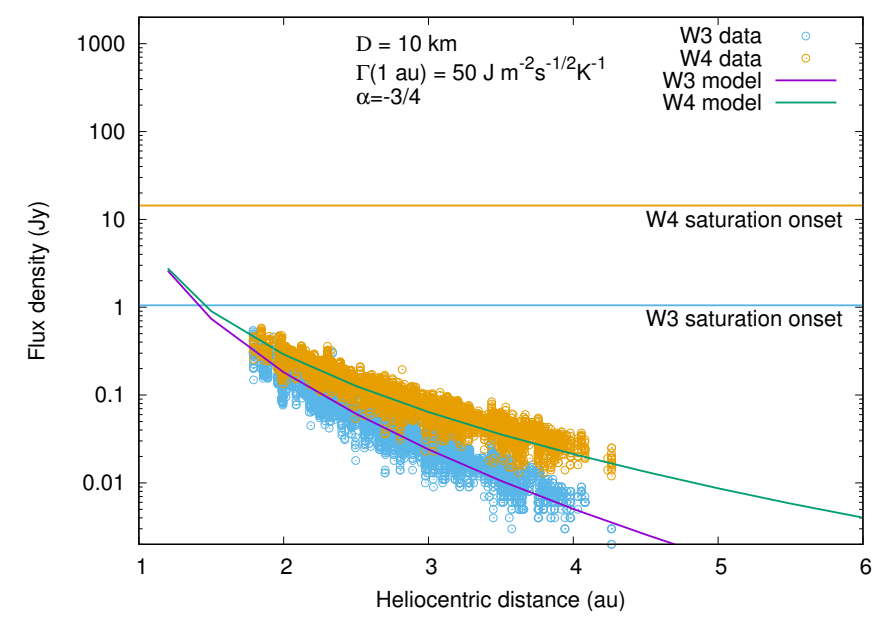

Fig. 6. Same as Fig. 5 but with $\alpha=-3 / 4$ and $\Gamma_{0}=50 \mathrm{~J} \mathrm{~m}^{-2} \mathrm{~s}^{-1 / 2} \mathrm{~K}^{-1}$.

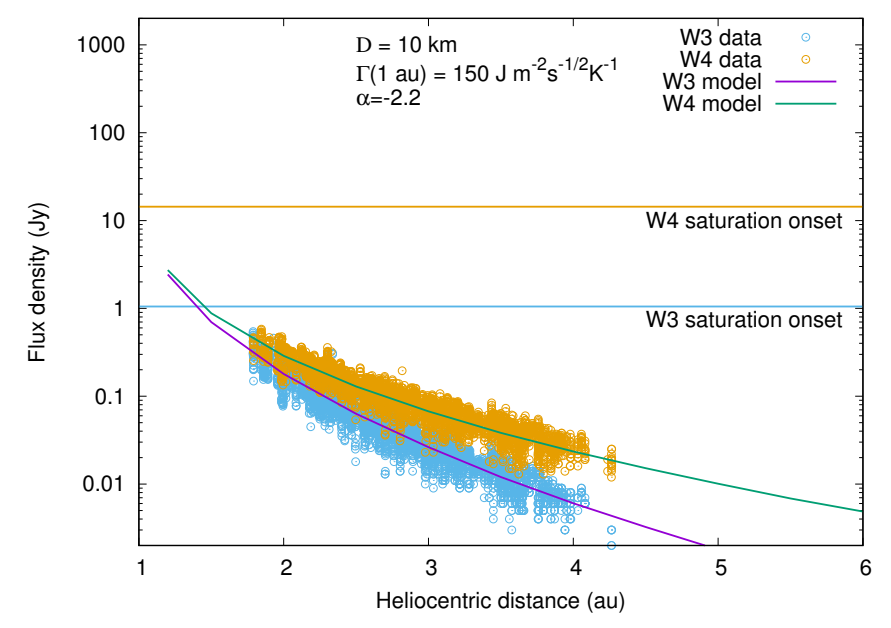

Fig. 7. Same as Fig. 5 but with $\alpha=-2.2$ and $\Gamma_{0}=150 \mathrm{~J} \mathrm{~m}^{-2} \mathrm{~s}^{-1 / 2} \mathrm{~K}^{-1}$.

and a finer and more complete sampling of $\Gamma, \alpha$, and $P$, it might be possible to obtain a model with a high "goodness of fit" (see e.g. Press et al. 1986).

We have not considered the completeness of the samples and we emphasise that the database does not currently contain the full WISE catalogue, but only the data pertaining to those objects that are featured in the AKARI catalogue. The AKARI catalogue is complete in the Main Belt down to sizes $20 \mathrm{~km}$ (Usui et al. 2013, 2014), but contains fewer Hildas/Cybeles/Jupiter Trojans in the L18W band. Also, in future works, we will incorporate the full WISE catalogue into the database to have more NEAs and members of populations beyond the main belt.

\section{Future updates of the SBNAF Infrared Database}

\subsection{Planned updates}

A full assessment of all Herschel/PACS main belt asteroid observations were performed in the framework of the Small Bodies: Near and Far Horizon 2020 project (Müller et al. 2018). Farinfrared flux densities obtained from these measurements will be made public with the next release of the database. Infrared data from serendipitous observations of bright main belt asteroids with the Herschel/PACS and SPIRE photometer detectors are also planned to be added. 

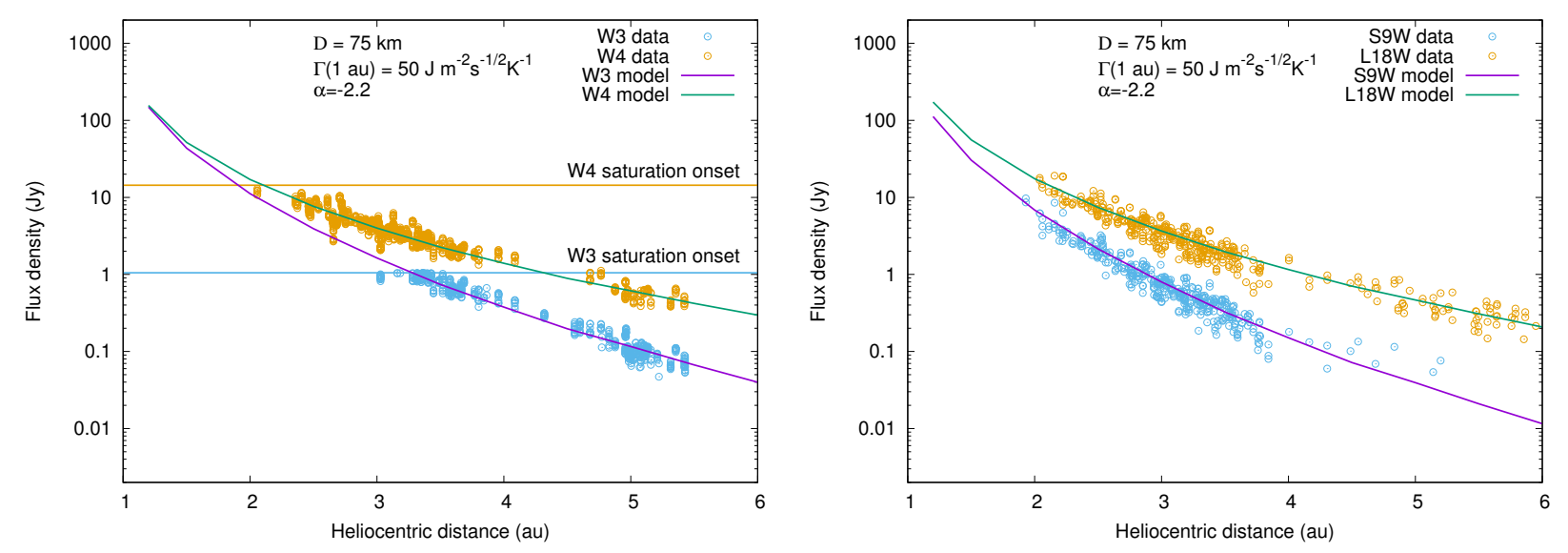

Fig. 8. Left: the onset of partial saturation limits the usefulness of the W3 data for the 75-km population. The gap between the main belt and the Hildas/Cybeles/Jupiter Trojans is apparent. Right: same plot but using AKARI data. In this case there were no saturation limits but there were fewer $18-\mu \mathrm{m}$ data at $r>4$ au.

\subsection{Submitting data}

Researchers who have infrared measurements can submit their reduced data to the SBNAF Infrared Database by sending an email to the address irdb@csfk.mta.hu. After the submission the data will be processed as it was described in sections above, and these processed and auxiliary data will be uploaded to the database. We request the input data in a CSV file (simple attachment of the cover email), with the following columns:

- Number: if possible, asteroid number, e.g. 275809.

- Designation: provisional designation, e.g. 2001 QY297.

- Observatory/project: name of the observatory or the space mission, e.g. Herschel, WISE, etc.

- Observatory_code: JPL/Horizons code of the observatory or spacecraft, e.g.500@-486.

- Instrument_detector: instrument of the observatory or spacecraft used in the specific measurement.

- Obsmode: observation mode of the instrument, if available.

- Observation_IDs: observation ID of the measurement, if available (e.g. AORKEY in the case of Spitzer, and OBSID in the case of Herschel measurements).

- Start: start time of the measurement in Julian date format.

- End: end time of the measurement in Julian date format.

- Band or filter: name of the filter or band used for the measurement.

- Calibrated_inband_flux: in-band photometric flux density in [Jansky] units, with all photometric corrections applied, including aperture or encircled energy fraction corrections, but without colour correction.

- Inband_flux_error: uncertainty of the in-band photometric flux density "calibrated_inband_flux", with all direct photometric errors considered, but without errors related to the spectral energy distribution of the target (colour correction), and also without the consideration of the absolute photometric error of the instrument.

- Comments: comments regarding the measurement; e.g. the flux density is an upper limit, the flux is already colour corrected, or it is obtained from a combination of more than one measurements, etc.

Acknowledgements. This work has dedicatedly been supported by European Union's Horizon 2020 Research and Innovation Programme, under Grant Agreement no 687378 ("Small Bodies: Near and Far"). The operation of the database is supported by the Hungarian Research, Development and Innovation Office (grant no. K-125015 and PD-128360), and by the Hungarian Academy of
Sciences (EUHUNKPT/2018). This research made use of Astropy (http:// wWw . astropy.org), a community-developed core Python package for Astronomy Astropy Collaboration $(2013,2018)$. We sincerely thank the referee for the thorough and detailed review.

\section{References}

Alí-Lagoa, V., Müller, T. G., Usui, F., \& Hasegawa, S. 2018, A\&A, 612, A85 Archinal, B., A'Hearn, M. F., Bowell, E., et al. 2011, CeMDA, 109, 101 Astropy Collaboration (Robitaille, T. P., et al.) 2013, A\&A, 558, A33 Astropy Collaboration (Price-Whelan, A. M., et al.) 2018, AJ, 156, 123 Balog, Z., Müller, T. G., Nielbock, M., et al. 2014, Exp. Astron., 37, 129 Bottke, Jr., W. F., Vokrouhlický, D., Rubincam, D. P., \& Nesvorný, D. 2006, Ann. Rev. Earth Planet. Sci., 34, 157

Bowell, E. G., Hapke, B., Domingue, D., et al. 1989, in Application of Photometric Models to Asteroids, eds. T. Gehrels, M. T. Matthews, \& R. P. Binzel (Tucson: University of Arizona Press), Asteroids II, 524

Brucker, M. J., Grundy, W. M., \& Stansberry, J. A. 2009, Icarus, 201, 284

Cutri, R. M., Wright, E. L., Conrow, T., et al. 2012, Explanatory Supplement to the WISE All-Sky Data Release Products

Cutri, R. M., Wright, E. L., Conrow, T., et al. 2013, Explanatory Supplement to the WISE All-Sky Data Release Products

Delbó, M., \& Tanga, P. 2009, Planet. Space Sci., 57, 259

Delbó, M., Dell'Oro, A., Harris, A. W., et al. 2007, Icarus, 190, 236

Delbó, M., Libourel, G., Wilkerson, J., et al. 2014, Nature, 508, 233

Delbó, M., Mueller, M., Emery, J. P., Rozitis, B., \& Capria, M. T. 2015, in Asteroid Thermophysical Modeling (Tucson: University of Arizona Press), Asteroids IV, 89

Delbó, M., Walsh, K., Bolin, B., et al. 2017, Science, 357, 1026

Duffard, R., Pinilla-Alonso, N., Santos-Sanz, P., et al. 2014, A\&A, 564, A92

Durech, J., Carry, B., Delbó, M., et al. 2015, in Asteroid Models from Multiple Data Sources (Tucson: University of Arizona Press), Asteroids IV, 895, 183 Durech, J., Delboó, M., Carry, B., et al. 2017, A\&A, 604, A27

Egan, M. P., Price, S. D., Kraemer, K. E., et al. 2003, Air Force Research Laboratory Technical Report No. AFRL-VS-TR-2003-1589

Enos, H. L., \& Lauretta, D. S. 2019, Nat. Astron., 3, 363

Erard, S., Cecconi, B., \& Le Sidaner, P. 2018, Planet. Space Sci., 150, 65

Fornasier, S., Lellouch, E., Müller, T., et al. 2013, A\&A, 555, A15

Hanuš, J., Delbó, M., D̆urech, J., \& Alí-Lagoa, V. 2015, Icarus, 256, 101

Hanuš, J., Delbó, M., Durech, J., \& Alí-Lagoa, V. 2018, Icarus, 309, 297

Harris, A. W., Mommert, M., \& Hora, J. L. 2011, AJ, 141, 75

Hasegawa, S., Müller, T. G., Kuroda, D., Takita, S., \& Usui, F. 2013, PASJ, 65, 34

Jakosky, B. M. 1986, Icarus, 66, 117

Keihm, S. J. 1984, Icarus, 60, 568

Keihm, S., Tosi, F., \& Kamp, L. 2012, Icarus, 221, 395

Kiss, C., Szabó, Gy., Horner, J., et al. 2013, A\&A, 555, A3

Kopp, G., \& Lean, J. L. 2011, Geophys. Res. Lett., 38, L01706

Kuhrt, E., \& Giese, B. 1989, Icarus, 81, 102

Lagerros, J. S. V. 1996, A\&A, 310, 1011

Lagerros, J. S. V. 1998, A\&A, 332, 1123

Lellouch, E., Santos-Sanz, P., Lacerda, P., et al. 2013, A\&A, 557, A60 
Lellouch, E., Santos-Sanz, P., Fornasier, S., et al. 2016, A\&A, 588, A2 Lim, T. L., Stansberry, J., Müller, T. G., et al. 2010, A\&A, 518, L148 Mainzer, A., Bauer, J., Grav, T., et al. 2011, ApJ, 731, 53

Mainzer, A., Usui, F., \& Trilling, D. E. 2015, in Space-Based Thermal Infrared Studies of Asteroids (Tucson: University of Arizona Press), Asteroids IV, 895, 89

Marciniak, A., Bartczak, P., Müller, T., et al. 2018a, A\&A, 610, A7

Marciniak, A., Ali-Lagoa, V., Müller, T., et al. 2018b, A\&A, 625, A139

Marsset, M., Carry, B., Dumas, C., et al. 2017, A\&A, 604, A64

Masiero, J., Mainzer, A., Grav, T., et al. 2011, ApJ, 741, 68

Mill, J. D. 1994, in Midcourse Space Experiment (MSX): An Overview of the Instruments and Data Collection Plans, Proc. SPIE, 2232, 200

Mill, J. D., O’Neil, R. R., Price, S., et al. 1994, J. Spacecr. Rockets, 31, 900

Mommert, M., Harris, A. W., Kiss, C., et al. 2012, A\&A, 541, A93

Mueller, M., Harris, A. W., Bus, S. J., et al. 2006, A\&A, 447, 1153

Müller, T. G. 2002, Meteorit. Planet. Sci., 37, 1919

Müller, T. G., \& Lagerros, J. S. V. 1998, A\&A, 338, 340

Müller, T. G., Lellouch, E., Böhnhardt, H., et al. 2009, Earth Moon Planets, 105, 209

Müller, T. G., Lellouch, E., Stansberry, J., et al. 2010, A\&A, 518, L146

Müller, T. G., O’Rourke, L., Barucci, A. M., et al. 2012, A\&A, 548, A36

Müller, T. G., Miyata, T., Kiss, C., et al. 2013, A\&A, 558, A97

Müller, T. G., Kiss, C., Scheirich, P., et al. 2014a, A\&A, 566, A22

Müller, T. G., Balog, Z., Nielbock, M., et al. 2014b, Exp. Astron., 37, 253

Müller, T. G., Hasegawa, S., \& Usui, F. 2014c, PASJ, 66, 5217

Müller, T. G., Durech, J., \& Ishiguro, M. 2017, A\&A, 599, A103

Müller, T. G., Marciniak, A., Kiss, C., et al. 2018, AdSpR, 62, 2326

Müller, T. G., Kiss, C., Ali-Lagoa, V., et al. 2019, Icarus, 334, 39

Müller, T. G., Lellouch, E., \& Fornasier, S. 2020, in Trans-Neptunian Objects and Centaurs at Thermal Wavelengths, eds. D. Prialnik, M. A. Barucci, \& L. Young, The Transneptunian Solar System, 153

Murakami, H., Baba, H., Barthel, P., et al. 2007, PASJ, 59, S369

Neugebauer, G., Habing, H. J., van Duinen, R., et al. 1984, ApJ, 278, 1

Nielbock, M., Müller, T. G., Klaas, U., et al. 2013, Exp. Astron., 36, 631

Ochsenbein, F., Bauer, P., \& Marcout, J. 2000, A\&AS, 143, 23

O’Rourke, L., Müller, T. G., Valtchanov, I., et al. 2012, Planet. Space Sci., 66, 192

Oszkiewicz, D. A., Muinonen, K., Bowell, E., et al. 2011, J. Quant. Spectrosc. Radiat. Transf., 112, 1919

Ortiz, J. L., Santos-Sanz, P., Sicardy, B., et al. 2017, Nature, 550, 219
Pál, A., Kiss, C., Müeller, T. G., et al. 2012, A\&A, 541, L6

Pál, A., Kiss, Cs., Horner, J., et al. 2015, A\&A, 583, A93

Pilbratt, G. L., Riedinger, J. R., Passvogel, T., et al. 2010, A\&A, 518, L1

Poglitsch, A., Waelkens, C., Geis, N., et al. 2010, A\&A, 518, L2

Press, W. H., Flannery, B. P., \& Teukolsky, S. A. 1986, Numerical Recipes. The Art of Scientific Computing (Cambridge: University Press)

Price, S. D., \& Witteborn, F. C. 1995, in MSX Mission Summary, eds. M. R. Haas, J. A. Davidson, \& E. F. Erickson, ASP Conf. Ser., 73, 685

Price, S. D., Tedesco, E. F., Cohen, M., et al. 1998, in Astronomy on the Midcourse Space Experiment, eds. B. J. McLean, D. A. Golombek, J. J. E. Hayes, \& H. E. Payne (Dordrecht: Kluwer Academic Publishers), New Horizons from Multi-Wavelength Sky Surveys, Proceedings of the 179th Symposium of the International Astronomical Union, held in Baltimore, USA August 26-30, 1996, 115

Price, S. D., Egan, M. P., Carey, S. J., Mizuno, D. R., \& Kuchar, T. A. 2001, AJ, 121,2819

Rozitis, B., MacLennan, E., \& Emery, J. P. 2014, Nature, 512, 174

Rozitis, B., Green, S. F., MacLennan, E., \& Emery, J. P. 2018, MNRAS, 477, 1782

Santos-Sanz, P., Lellouch, E., Fornasier, S., et al. 2012, A\&A, 541, A92

Santos-Sanz, P., Lellouch, E., Groussin, O., et al. 2017, A\&A, 604, A95

Stansberry, J., Grundy, W., Brown, M., et al. 2008, in Physical Properties of Kuiper Belt and Centaur Objects: Constraints from the Spitzer Space Telescope (Tucson: University of Arizona Press), The Solar System Beyond Neptune, 592, 161

Tedesco, E. F., Noah, P. V., Noah, M., \& Price, S. D. 2002a, AJ, 123, 1056

Tedesco, E. F., Egan, M. P., \& Price, S. D. 2002b, AJ, 124, 583

Trilling, D. E., Mueller, M., Hora, J. L., et al. 2010, AJ, 140, 770

Usui, F., Kuroda, D., Müller, T. G., et al. 2011, PASJ, 63, 1117

Usui, F., Kasuga, T., Hasegawa, S., et al. 2013, ApJ, 762, 56

Usui, F., Hasegawa, S., Ishiguro, M., et al. 2014, PASJ, 66, 5611

Vereš, P., Jedicke, R., Fitzsimmons, A., et al. 2015, Icarus, 261, 34

Vilenius, E., Kiss, C., Mommert, M., et al. 2012, A\&A, 541, A94

Vilenius, E., Kiss, C., Müller, T. G., et al. 2014, A\&A, 564, A35

Vilenius, E., Stansberry, J., Müller, T. G., et al. 2018, A\&A, 618, A136

Vokrouhlický, D., Bottke, W. F., Chesley, S. R., Scheeres, D. J., \& Statler, T. S. 2015, in The Yarkovsky and YORP Effects, eds. P. Michel, F. E. DeMeo, \& W. F. Bottke (Tucson: University of Arizona Press), Asteroids IV, 509

Watanabe, S.-I., Tsuda, Y., Yoshikawa, M., et al. 2017, Space Sci. Rev., 208, 3 Wright, E. L., Eisenhardt, P. R. M., \& Mainzer, A. K. 2010, AJ, 140, 1868 


\section{Appendix A: Summary of database fields}

Below we give a description of the output fields of the Infrared Database. The unit and the data type of the specific field are given in squared and regular brackets, respectively.

naifid. NASA's Navigation and Ancillary Information Facility $^{11}$ solar system object code of the target (LONG).

targetname. The name or designation of the asteroid. (STRING).

observatory_project. Name of the observatory/ space mission (STRING). The possible values are: "IRAS": Infrared Astronomy Satellite; "AKARI": Akari Space Telescope; "MSX": Midcourse Space Experiment; "WISE": Wide-field Infrared Survey Explorer; "HSO": Herschel Space Observatory.

See Table 1 for a list of instruments, filters and observatory codes used in this database. References for the listed instruments and flux density measurements can be found in Sect. 2.2.

observatory_code. JPL/Horizons code of the observatory/spacecraft, see Table 1 for a list (STRING).

instrument_detector. Instrument of the observatory/spacecraft used in that specific measurement, see Table 1 for a list (STRING) (STRING).

obsmode. Observation mode, also listed in Table 1. For a descrition of the observing modes, see the respective references of the instruments in Sect. 2.2. For instruments working in survey mode (like IRAS and MSX) with which no pointed observations were possible and data are taken from the survey data "survey" in the "obsmode" column indicates the default observing mode (STRING).

observation_IDs. Mission-specific identifier of the observation, e.g. OBSID for Herschel measurements and AORKEY for Spitzer observations [unitless] (STRING).

observation_start_time. Start time of the measurement [Julian date] (DOUBLE).

observation_mid_time. Mid-time of the measurement [Julian date] (DOUBLE).

observation_end_time. End time of the measurement [Julian date] (DOUBLE).

datetime. Observation date in the format "YYYY:MM:DD hh:mm:ss.sss", with YYYY: year; MM: month in string format (Jan., Feb., etc.), DD: day of the month; hh: hour of the day; mm: minutes; ss.sss: seconds with three-digit accuracy (STRING).

band_filter. Name of the filter/band used for the specific observation (STRING).

calibrated_inband_flux_Jy. In-band photometric flux density in [Jansky] units, with all photometric corrections applied, including aperture/encircled energy fraction corrections, but without colour correction (DOUBLE). Sources of original data are listed in Sect. 2.2.

inband_flux_error_Jy. Uncertainty of the in-band photometric flux density “calibrated_inband_flux_Jy", with all direct photometric errors considered, but without errors related to the spectral energy distribution of the target (colour correction), and also without the consideration of the absolute photometric error of the instrument (DOUBLE).

quality_flags. (STRING)

\footnotetext{
${ }^{11}$ https://naif.jpl.nasa.gov/pub/naif/toolkit_docs/
} FORTRAN/req/naif_ids.html
- WISE ${ }^{12}$ :

- Contamination and confusion flags:

- P - Persistence. Source may be a spurious detection of $(\mathrm{P})$.

- $\mathrm{p}$ - Persistence. Contaminated by (p) a short-term latent image left by a bright source.

- 0 (number zero) - Source is unaffected by known artifacts.

- Photometric quality flags:

- A - Source is detected in this band with a flux signal-to-noise ratio $\mathrm{w} \_s n r>10$.

- $\mathrm{B}$ - Source is detected in this band with a flux signalto-noise ratio $3<\mathrm{w} \_$snr $<10$.

orbital_param_A. semi-major axis of the target's orbit, as obtained from JPL/Horizons [AU] (DOUBLE).

orbital_param_EC. eccentricity of the target's orbit, as obtained from JPL/Horizons [unitless] (DOUBLE).

orbital_param_IN. inclination of the target's orbit, as obtained from JPL/Horizons [deg] (DOUBLE).

orbital_param_OM. longitude of the ascending node of the target's orbit, as obtained from JPL/Horizons [deg] (DOUBLE).

orbital_param_W. argument of the periapsis of the target's orbit, as obtained from JPL/Horizons [deg] (DOUBLE).

orbital_param_MA. mean anomaly of the target's orbit, as obtained from JPL/Horizons [deg] (DOUBLE).

absolute_magnitude_H. absolute magnitde of the target, i.e. the visual magnitude an observer would record if the asteroid were placed $1 \mathrm{AU}$ away, and $1 \mathrm{AU}$ from the Sun and at a zero phase angle, as obtained from JPL/Horizons [mag] (FLOAT).

slope_parameter_G. " $G$ " slope parameter of the target, describing the dependence of the apparent brightness on the phase angle (light scattering on the asteroid's surface); for more details, see Bowell et al. (1989) (FLOAT). Note that the default slope parameter in the Horizons system is the canonical $G=0.15$, also used as a generally accepted value in applications when specific values are note available (see e.g. ExploreNEO, Trilling et al. 2010; Harris et al. 2011).

jpl_obj_radius. estimated radius of the target as obtained from JPL Horizons [km] (FLOAT).

jpl_obj_albedo. estimated V-band geometric albedo of the target as obtained from JPL Horizons (FLOAT).

Right_Ascension_RA. right ascension (J2000) of the target at observation mid-time, calculated from the orbit by JPL/Horizons [deg] (FLOAT).

Declination_DEC. declination (J2000) of the target at observation mid-time, calculated from the orbit by JPL/Horizons [deg] (FLOAT).

$R A$ rate. rate of change in right ascension [arc$\operatorname{sec~s}^{-1} \equiv \operatorname{deg~} \mathrm{h}^{-1}$ ] (FLOAT).

DEC_rate. rate of change in declination [arc$\operatorname{sec~s}^{-1} \equiv \overline{\operatorname{deg~h}}{ }^{-1}$ ] (FLOAT).

apparent_magnitude_V. estimated apparent brightness of the target in V-band at observation mid-time, as obtained by JPL/Horizons [mag] (FLOAT).

\footnotetext{
12 http://wise2.ipac.caltech.edu/docs/release/allsky/ expsup/index.html
} 
heliocentric_distance_r. heliocentric distance of the target at observation mid-time, as obtained by JPL/Horizons [AU] (DOUBLE).

obscentric distance delta. observer to target distance at observation mid-time, as obtained by JPL/Horizons [AU] (DOUBLE).

lighttime. elapsed time since light (observed at print-time) would have left or reflected off a point at the center of the target [s] (FLOAT).

solar_elongation_elong. target's apparent solar elongation seen from the observer location at print-time, in degrees (FLOAT).

before_after_opposition. flag regarding the target's apparent position relative to the Sun in the observer's sky. " $T$ " indicates trailing, "L" leading position with respect to the Sun (STRING).

phase angle alpha. Sun-Target-Observer angle at observation mid-time, as obtained by JPL/Horizons [deg] (FLOAT).

ObsEcILon/ObsEclLat. observer-centered Earth ecliptic-ofdate longitude and latitude of the target center's apparent position, adjusted for light-time, the gravitational deflection of light and stellar aberration, in degrees, as obtained by JPL/Horizons [deg] (FLOAT).

target_[X,Y,Z]@sun. Sun-centered $X, Y, Z$ Cartesian coordinates of the target body at observation mid-time, in the reference frame defined in Archinal et al. (2011) [AU]. (DOUBLE).

target_[X,Y,Z]_@observer. observer-centered $X, Y, Z$ Cartesian coordinates of the target body at observation mid-time, in the reference frame defined in Archinal et al. (2011) [AU]. (DOUBLE).

observer_X_@sun. Sun-centered X,Y,Z Cartesian coordinates of the observer at observation mid-time, in the reference frame defined in Archinal et al. (2011) [AU]. (DOUBLE) (DOUBLE).

reference_wavelengths_micron. reference wavelength of the measuring filter in $[\mu \mathrm{m}]$ units (FLOAT). colour_correction_factor.colour correction factor applied to obtain monochromatic flux density from in-band flux density [unitless]; see Sect. 3.2 for details (FLOAT).

colour corrected flux density. monochromatic flux density (colour corrected in-band flux density) [Jy]; see Sect. 3.2 for details (DOUBLE).

absolute_flux_error. absolute uncertainty of the monochromatic flux density including the uncertainty of the absolute flux calibration [Jy]; see Sect. 3.2 for details (DOUBLE).

comments_remarks. comments regarding the quality of the measurement/information when an assumed value was applied in the calculations; e.g. indicating whether the target is (also) regarded as a comet in JPL/Horizons; non standard value of geometric albedo is also marked here when it is not taken from JPL/Horizons (STRING).

LTcorrected_epoch. The lighttime corrected epoch, calculated as observation_mid_time - lighttime/3600./24. [day] (DOUBLE).

documents_references. publications and resources which the photometric data were taken from (STRING). A list of codes can be found in Sect. 2.2.

input_table_source. name of the input file which the database was generated from (strictly for internal usage) (STRING).

data last modification. date when the record was last modified, in "human readable" format: "YYYY-MMM-DD hh:mm:ss" (STRING).

alt_target_name. possible alternative names for the object, like asteroid number and provisional designation. (STRING).

\section{Appendix B: Example of infrared database fields}

Table B. 1 presents the specification of the infrared database fields through an example (Akari measurement of (25 143) Itokawa) as it was at the time of the production of this document. Changes may apply and the description should be taken from the latest version of the file AstIrDbTbl_keys_v<version_number >.txt. 
Table B.1. Example of infrared database fields.

\begin{tabular}{|c|c|c|c|c|}
\hline Parameter & Type & Unit & Example value & $\begin{array}{l}\text { Retrieved } \\
\text { from JPL }\end{array}$ \\
\hline 01 naifid & LONG & _- & 2025143 & No \\
\hline 02 targetname & STRING & - & Itokawa & No \\
\hline 03 observatory_project & STRING & - & AKARI & No \\
\hline 04 observatory_code & STRING & - & $500 @ 399$ & No \\
\hline 05 instrument_detector & STRING & - & IRC-MIR-L & No \\
\hline 06 obsmode & STRING & - & IRC02 & No \\
\hline 07 observation_IDs & STRING & - & & No \\
\hline 08 observation_start_time & DOUBLE & days & 2454308.04699 & No \\
\hline 09 observation_mid_time & DOUBLE & days & 2454308.04699 & No \\
\hline 10 observation_end_time & DOUBLE & days & 2454308.04699 & No \\
\hline 11 datetime & STRING & - & 2007-Jul.-26 13:07:40.000 & No \\
\hline 12 band_filter & STRING & - & L15 & No \\
\hline 13 calibrated_inband_flux_Jy & DOUBLE & Jy & 0.02 & No \\
\hline 14 inband_flux_error_Jy & DOUBLE & Jy & 0.001 & No \\
\hline 15 quality_flags & STRING & - & & No \\
\hline 16 orbital_param_A & DOUBLE & au & 1.32404449 & Yes \\
\hline 17 orbital_param_EC & DOUBLE & - & 0.28018482 & Yes \\
\hline 18 orbital_param_IN & DOUBLE & deg & 1.62206524 & Yes \\
\hline 19 orbital_param_OM & DOUBLE & $\operatorname{deg}$ & 69.09531692 & Yes \\
\hline 20 orbital_param_W & DOUBLE & $\operatorname{deg}$ & 162.77163687 & Yes \\
\hline 21 orbital_param_MA & DOUBLE & deg & 32.28481921 & Yes \\
\hline 22 absolute_magnitude_H & FLOAT & mag & 19.2 & Yes \\
\hline 23 slope_parameter_G & FLOAT & - & 0.15 & Yes \\
\hline 24 jpl_obj_radius & FLOAT & $\mathrm{km}$ & 0.165 & Yes \\
\hline 25 jpl_obj_albedo & FLOAT & - & 0.1 & Yes \\
\hline 26 Right_Ascension_RA & FLOAT & $\operatorname{deg}$ & 209.5522 & Yes \\
\hline 27 Declination_Dec & FLOAT & $\operatorname{deg}$ & -16.11449 & Yes \\
\hline 28 RA_rate & FLOAT & "/s & 0.0654038 & Yes \\
\hline 29 Dec_rate & FLOAT & "/s & -0.028018 & Yes \\
\hline 30 apparent_magnitude_V & FLOAT & mag & 19.16 & Yes \\
\hline 31 heliocentric_distance_r & DOUBLE & au & 1.05402381 & Yes \\
\hline 32 obscentric_distance_delta & DOUBLE & au & 0.28128128 & Yes \\
\hline 33 lighttime & FLOAT & $\mathrm{s}$ & 140.3607 & Yes \\
\hline 34 solar_elongation_elong & FLOAT & $\operatorname{deg}$ & 90.0359 & Yes \\
\hline 35 before_after_opposition & STRING & - & $/ \mathrm{T}$ & Yes \\
\hline 36 phase_angle_alpha & FLOAT & $\operatorname{deg}$ & -74.49 & Yes \\
\hline 37 ObsEclLon & FLOAT & deg & 213.2239 & Yes \\
\hline 38 ObsEclLat & FLOAT & deg & -3.795164 & Yes \\
\hline 39 target_X_@sun & DOUBLE & au & 0.319379555249375 & Yes \\
\hline 40 target_Y_@sun & DOUBLE & au & -1.00430258867518 & Yes \\
\hline 41 target_Z_@sun & DOUBLE & au & -0.0185976638442141 & Yes \\
\hline 42 target_X_@observer & DOUBLE & au & -0.235047951829334 & Yes \\
\hline 43 target_Y_@observer & DOUBLE & au & -0.153329693108554 & Yes \\
\hline 44 target_Z_@observer & DOUBLE & au & -0.0186135080544228 & Yes \\
\hline 45 observer_X_@sun & DOUBLE & au & 0.554427507078709 & Yes \\
\hline 46 observer_Y_@sun & DOUBLE & au & -0.850972895566624 & Yes \\
\hline 47 observer_Z_@sun & DOUBLE & au & $1.58442102086986 \mathrm{E}-05$ & Yes \\
\hline 48 reference_wavelengths_micron & FLOAT & micron & 24.0 & No \\
\hline 49 colour_correction_factor & FLOAT & - & 0.984 & No \\
\hline 50 colour_corrected_flux_density & DOUBLE & Jy & 0.021 & No \\
\hline 51 absolute_flux_error & DOUBLE & Jy & 0.001 & No \\
\hline 52 comments_remarks & STRING & - & $\begin{array}{l}\text { An assumed geometric albedo of } 0.1 \text { was used to } \\
\text { calculate the colour-correction factor }\end{array}$ & No \\
\hline 53 LTcorrected_epoch & DOUBLE & days & $\begin{array}{l}\text { 2454308.045366/to be calculated from } \\
\text { (observation_mid_time - lighttime/3600./24.) }\end{array}$ & No \\
\hline 54 documents_references & STRING & - & Müller et al. (2014a,b,c); AKARIAFC & No \\
\hline 55 date_last_modification & STRING & - & 2018-08-31 15:13:25 & No \\
\hline 56 alt_target_name & STRING & - & 25143\#1998 SF36\#2025143 & No \\
\hline
\end{tabular}

